\title{
Spectroscopy of polycyclic aromatic hydrocarbons and very small grains in photodissociation regions
}

\author{
M. Rapacioli ${ }^{1}$, C. Joblin ${ }^{1}$, and P. Boissel ${ }^{2}$ \\ ${ }^{1}$ Centre d'Étude Spatiale des Rayonnements, CNRS-Université Paul Sabatier - Observatoire Midi-Pyrénées, \\ 9 avenue du Colonel Roche, 31028 Toulouse, France \\ e-mail: mathias.rapacioli@cesr.fr \\ ${ }^{2}$ Laboratoire de Chimie Physique, CNRS-Université Paris Sud, Bât. 350, 91405 Orsay Cedex, France
}

\section{Received 6 May 2004 / Accepted 5 August 2004}

\begin{abstract}
We have coupled a singular value decomposition method with a Monte Carlo search algorithm to analyse the midinfrared ISOCAM ${ }^{\star}$ spectral maps of photodissociation regions (PDRs) in NGC 7023 and $\rho$ Oph-SR3.Three different spectra and their associated spatial distribution were extracted from this analysis. It is shown that they can be associated with polycyclic aromatic hydrocarbons (PAHs) in their cationic and neutral forms and a third population of carbonaceous very small grains (VSGs). The method allows for the first time (i) to separate the contribution of neutral PAHs to the interstellar emission spectrum from that of cationic PAHs; (ii) to show that the $7.8 \mu \mathrm{m}$ component of the " $7.7 \mu \mathrm{m}$ " broad feature is carried by VSGs, whereas the $7.6 \mu \mathrm{m}$ component is due to PAHs; and (iii) to give evidence that free-flying PAHs are produced in PDRs by photoevaporation of VSGs. It is proposed that these carbonaceous VSGs are indeed PAH clusters. We derived a minimal size of 400 carbon atoms per cluster and estimated a UV absorption power of the order of $10^{-24} \mathrm{~W} \mathrm{C}^{-1}$ for their dissociation.
\end{abstract}

Key words. ISM: lines and bands - ISM: clouds - ISM: dust, extinction - methods: data analysis - astrochemistry

\section{Introduction}

Twenty years ago, Léger \& Puget (1984) and Allamandola et al. (1985) proposed that Polycyclic Aromatic Hydrocarbons (PAHs) are an important component of interstellar dust, accounting for the unidentified infrared (IR) emission bands at $3.3,6.2,7.7,8.6,11.3$ and $12.7 \mu \mathrm{m}$. These bands, also called Aromatic Infrared Bands (AIBs), have now been observed in many places where interstellar or circumstellar matter is submitted to UV irradiation, in our Galaxy as well as in external galaxies. Many observations were performed by the recent IR space missions, in particular the Infrared Space Observatory (ISO; Kessler et al. 1996) and the Infrared Telescope in Space (IRTS; Murakami et al. 1996).

The identification of interstellar PAH species has motivated a wealth of laboratory measurements (Szczepanski \& Vala 1993; Hudgins et al. 1994, 1995; Kurtz 1993; Joblin et al. 1995; Cook et al. 1998; Oomens et al. 2000; Kim et al. 2001 and more recently Banisaukas et al. 2004; Mattioda et al. 2003; Huneycutt et al. 2004). Many quantum chemical calculations have also been performed (de Frees et al. 1993; Pauzat et al. 1995; Langhoff 1996; Ellinger et al. 1999 and more recently

^ Based on observations with ISO, an ESA project with instruments funded by ESA Member States (especially the PI countries: France, Germany, The Netherlands and the UK) and with the participation of ISAS and NASA.
Hudgins et al. 2001; Pauzat \& Ellinger 2002; Bauschlicher 2002). Although these studies reinforce the idea that the emitters are PAHs most likely in their ionized form, none of the individual spectra nor their mixture could so far match the observed spectra. This is likely due to the specific nature of the interstellar species which depends on their formation mechanism and further environmental effects. In particular, the UV photons which excite the IR emission can lead not only to photoionization but also to photodissociation of PAHs.

Regions where UV photons dominate the photophysics and chemistry are called Photo-Dissociation Regions (PDRs). They are formed at the boundary between hot stars and nearby molecular clouds. In these regions, the UV flux and spectral distribution vary as one goes from the border of the cloud to the inner regions. It is therefore interesting to study how the mid-IR emission spectrum evolves as a function of the UV field properties. This study requires good spatial resolution since the UV field is rapidly affected by dust extinction and by radiative transfer in molecular lines. This was recently made possible thanks to the imagery and spectroscopy capabilities of the camera ISOCAM on board ISO (Cesarsky et al. 1996). Since the AIBs are relatively broad, the spectral resolution of the Circular Variable Filter $(\mathrm{CVF})$ mode $\left(\frac{\lambda}{\Delta \lambda}=50\right)$ was reasonably well adapted.

An important step in the data analysis consists of quantifying the mid-IR spectrum features in order to correlate the 
observed variations with the photochemical evolution of the emitting populations. Previous analysis of the AIB spectra involved a mathematical decomposition made of a polynomial continuum and of Lorentzian functions for the bands (Boulanger et al. 1998). Such a decomposition is useful to study the variations of the relative band strengths and of the band-to-continuum ratio within nebulae (Verstraete et al. 2001; Abergel et al. 2002). Boissel et al. (2001) proposed the use of a different approach in order to extract full spectral information for the involved chemical species. The authors showed that the Singular Value Decomposition (SVD) method can be applied to the ISOCAM-CVF data similarly to what is done in laboratory multicomponent analysis (Leger et al. 2002). In this approach, the spectrum derived for each pixel of the ISOCAM-CVF map is supposed to result from the superposition of a few elementary spectra which are associated with different chemical populations. The problem consists of determining the number of elementary chemical spectra and then to derive their spectral information.

This paper describes how a Monte Carlo search algorithm can be coupled with the SVD analysis to obtain these spectra. Compared to the previous paper (Boissel et al. 2001), we have avoided subjective criteria to select the best elementary spectra. To converge the method however requires a large number of pixels with a high signal-to-noise ratio. Nebulae with very high excitation conditions (Orion Bar, M 17,...) are excluded because of additional emitters such as ionized gas in the associated HII regions. Also, any photochemical evolution is expected to occur much faster and therefore at smaller angular scales. Our analysis is therefore limited to bright extended regions with mild UV excitation conditions. We present here the results obtained for the reflection nebula NGC 7023 and for the PDR surrounding the young stellar object SR3 in the $\rho$ Ophiuci molecular cloud.

After a short description of the studied objects (Sect. 2), we present our method in detail (Sects. 3 and 4). The spectra obtained with the SVD-Monte Carlo analysis and their spatial distributions are then shown (Sect. 5). The similarity between one of the spectra extracted from the SVD-Monte Carlo analysis and the spectrum observed in the object Ced 201 (Cesarsky et al. 2000) is pointed out as further validation of our decomposition. In Sect. 6, the extracted elementary spectra are attributed to different populations of PAH-like species and Very Small Grains (VSGs) and their relative spatial distributions are discussed qualitatively. Finally in Sect. 7, the UV field is calculated as a function of depth using a PDR numerical model. A size is derived for the observed population of VSGs as well as the characteristics of the UV field over which they are severly affected by photofragmentation.

\section{The observations}

The observations performed by ISOCAM in CVF mode are available in the ISO archive. We have selected two regions in which a bright star illuminates its parent cloud: HD 200775 and SR 3 in the reflection nebula NGC 7023 and in the $\rho$ Ophiuci ( $\rho$ Oph) cloud, respectively.
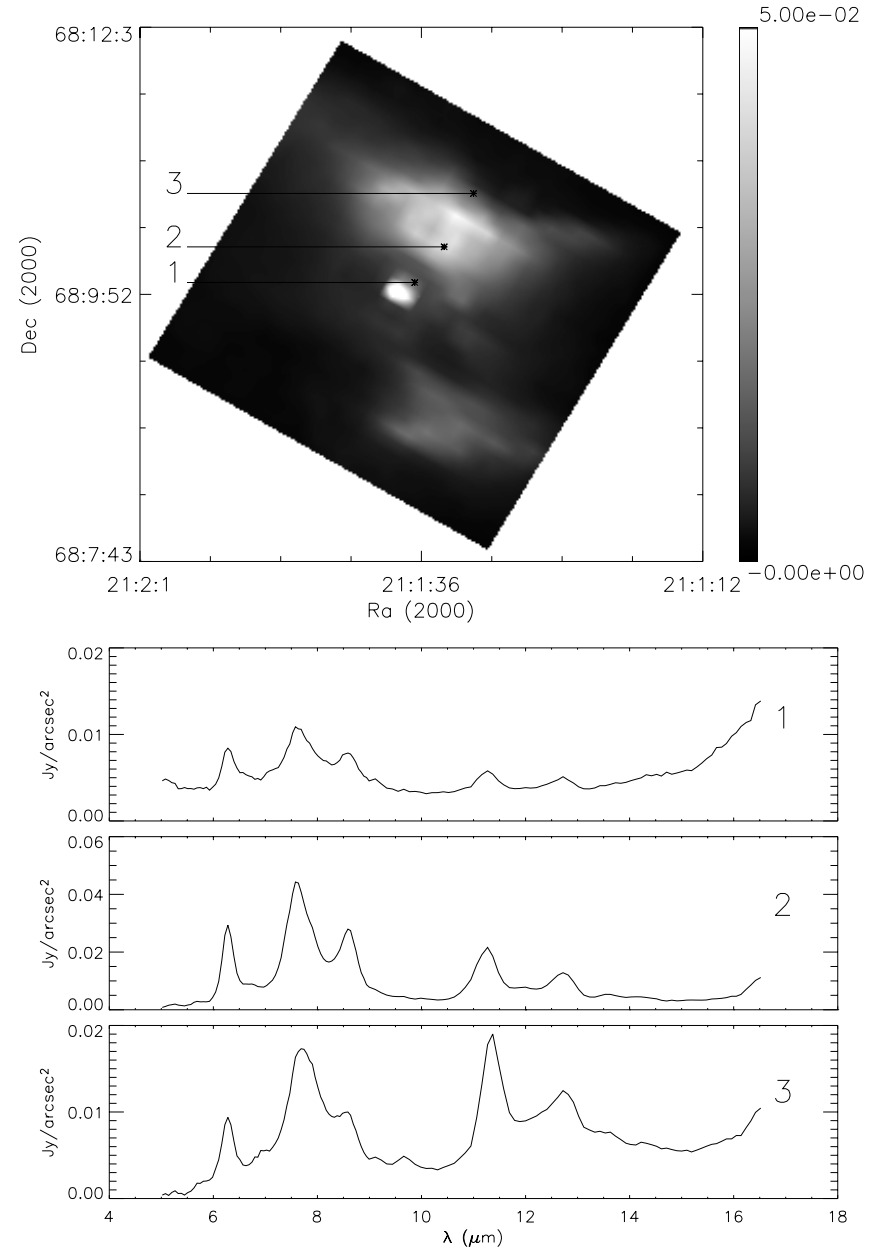

Fig. 1. ISOCAM map at $7.7 \mu \mathrm{m}$ of NGC $7023\left(\mathrm{Jy} / \operatorname{arcsec}^{2}\right)$ and three characteristic spectra extracted across the northern PDR.

\subsection{NGC 7023}

NGC 7023 is one of the most studied reflection nebulae. It is illuminated by a young B2.5 IVe star (Rogers et al. 1995), HD 200775, located at a distance of 430 pc (van den Ancker et al. 1997). Two PDRs are visible around the star (see Fig. 1). The brightest one lies at about $40^{\prime \prime}$ to the north-west of HD 200775 (Chokshi et al. 1988), the second one, less studied, is located $70^{\prime \prime}$ to the south of the star (Cesarsky et al. 1996). NGC 7023 has been observed in the radio (cf. for instance Rogers et al. 1995; Fuente et al. 1997; Gerin et al. 1998), in the IR (Sellgren et al. 1992; Lemaire et al. 1999; Martini et al. 1997; Laureijs et al. 1996; Cesarsky et al. 1996), and in the visible/UV (Witt et al. 1992; Federman et al. 1997). Emission from the AIBs is known to be present from combined ground-based and airplane-borned observations (Sellgren et al. 1985). ISOCAM has allowed spectroscopic studies of this object at rather higher spatial resolution (6" per pixel). To analyse the data, Boissel et al. (2001) applied the SVD method to the "Quick Look" product retrieved from the ISO archive (TDT Number 10702605). The data of higher quality we have used has been processed by Cesarsky et al. (1996).

Data at higher processing levels including the removal of the zodiacal and stray light emission were recently made 

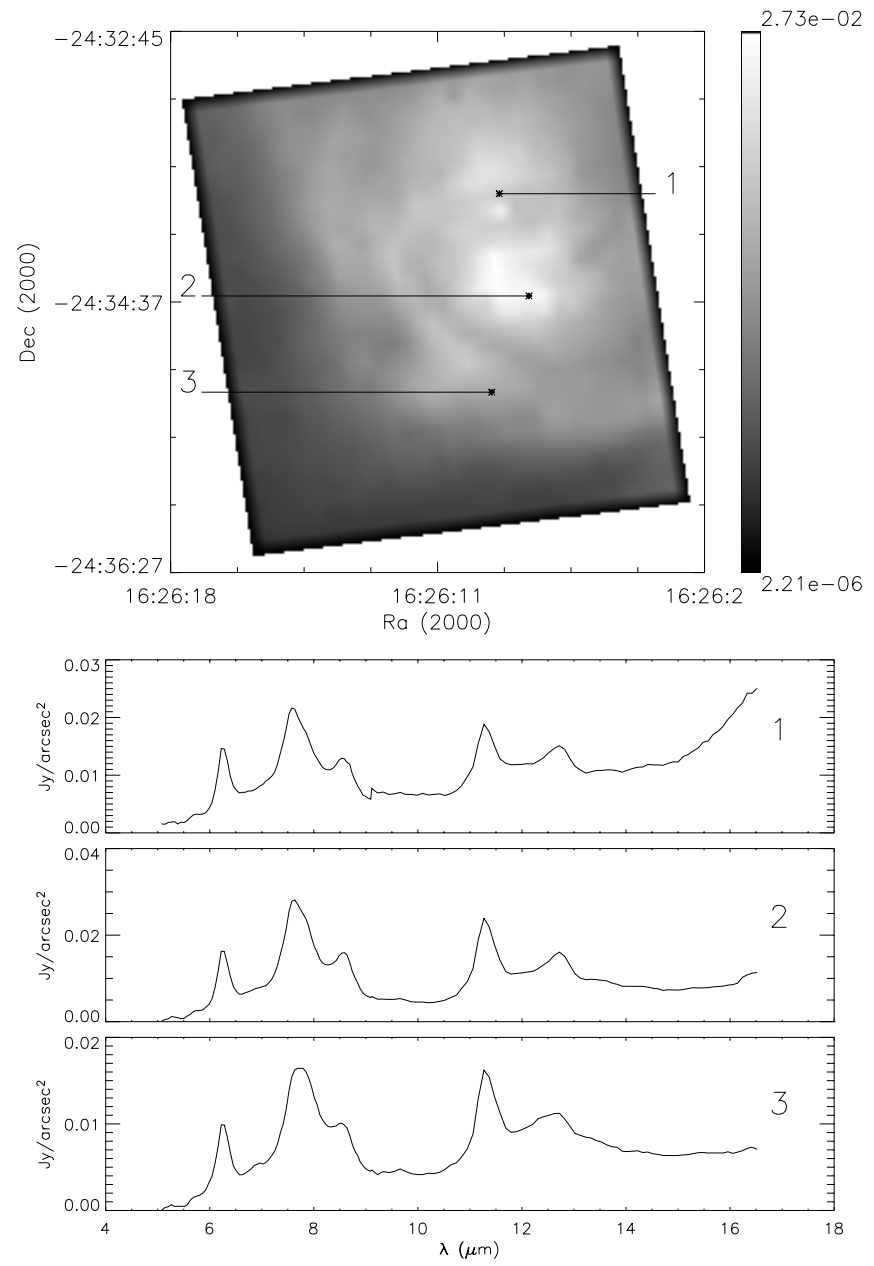

Fig. 2. ISOCAM map at $7.7 \mu \mathrm{m}$ in $\rho$ Oph $\left(\mathrm{Jy} / \operatorname{arcsec}^{2}\right)$ around the star SR 3 (point 1) and three characteristic spectra extracted within the nebula.

available by Boulanger et al. (2004) (the Highly Processed Data Products, HPDP). However, applying our mathematical analysis to this new set of data led to bad solutions as discussed in Sect. 4.3.

\section{2. $\rho$ Oph-SR3}

The $\rho$ Oph molecular cloud is one of the nearest cloud to the Sun. The PDR studied in the present paper is located in the main $\rho$ Oph cloud L1688 at a distance of about $140 \mathrm{pc}$ and results from the illumination of the molecular cloud by a bright embedded young star, SR3 of spectral type B7 (Bontemps et al. 2001). For this object, we were able to use the highly-processed data (Boulanger et al. 2004, TDT number 45801752 in the ISO archive).

\subsection{The data}

The ISOCAM CVF data on NGC 7023 and $\rho$ Oph-SR3 are shown in Figs. 1 and 2, with full spectral information between 5.15 and $16.5 \mu \mathrm{m}$ at the resolution of $\frac{\lambda}{\Delta \lambda}=50$ (Boulanger et al. 2004) and a pixel size of $66^{\prime \prime}$. Figures 1 and 2 illustrate the variations of the spectra within the nebulae: presence of a continuum at long wavelengths localised on the star (position 1), typical AIB spectra (position 2), presence of an underlying continuum inside the cloud (position 3). The band-to-continuum ratio is observed to decrease with increasing distance inside the cloud. We found that the spectra were of lower quality at the longest wavelengths and we therefore limited our analysis to the [5.2-15.9] $\mu \mathrm{m}$ spectral range.

\section{The singular value decomposition method}

The ISOCAM-CVF data are cubes of 32 pixels $\times 32$ pixels $\times$ 148 wavelengths whereas SVD is a mathematical decomposition which can only be applied to matrices. Since the spatial correlation is not a parameter of our analysis, it is possible to combine the data in a matrix $A$ of dimensions $m \times n$ ( $m=$ $32 \times 32=1024$ different spectra of $n=148$ wavelengths). The observed spectra are assumed to result from the linear combination of $k$ elementary chemical spectra with additional noise:

$A=C \times E_{k}+B$

where $E$ is the $k \times n$ matrix containing the $k$ elementary spectra, $C$ is the $m \times k$ matrix of weight coefficients which depends on the concentration of each species and the excitation conditions. $B$ is the $m \times n$ matrix of noise, which is assumed to be random noise (systematic instrumental errors are neglected). Because of noise, all the spectra contained in the matrix $A$ are linearly independent implying that the rank of the matrix $A$ is $n$. We define $A_{k}$ as the best reduced matrix of rank $k$ to fit $A$ (minimization of $\left.\left\|A-A_{k}\right\|\right)$. The product $C \times E_{k}$ can be considered as a good approximation of $A_{k}$ when $A$ contains a large number of spectra (large value of $m$ ). In that case, the subspace spanned by the spectra of $A_{k}$ approximates the subspace spanned by the spectra $E_{k}$. The matrix $A_{k}$ can be exactly calculated using the SVD method which decomposes $A$ as the product of three matrices.

$A=U \times S \times V$

where $U(m \times n)$ and $V(n \times n)$ are called the left and right singular matrices and $\mathrm{S}$ is the diagonal matrix of singular values sorted by decreasing order. $A_{k}$ is then calculated by truncating these matrices to the rank $k$ :

$A_{k}=U_{k} \times S_{k} \times V_{k}$

The subspace spanned by the spectra of $A_{k}$ is equivalent to the subspace spanned by $V_{k}$, which approximates the subspace spanned by $E_{k}$. The elementrary spectra $E_{k}$ can then be approximated by linear combinations of the eigenvectors $V_{k}$ :

$E_{k} \approx T \times V_{k}$

where $T$ is the $k \times k$ transformation matrix of rank $k$ between the mathematical spectra (eigenvectors) and the elementary chemical spectra. The determination of $T$ is described in the next section. An approximation of the weight matrix $C$ can then be derived from $T$ and the SVD matrices using

$A_{k}=(U \times S)_{k} \times V_{k} \approx(U \times S)_{k} \times T^{-1} \times E_{k}$ 
and

$A_{k} \approx C \times E_{k}$

which gives

$C \approx U_{k} \times S_{k} \times T^{-1}$.

In the following, we present the two steps which have been performed to extract the physical information:

(i) evaluation of the number $\mathrm{k}$ of chemical spectra;

(ii) calculation of the transformation matrix $T$ using a Monte Carlo algorithm.

\section{Extraction of physical information}

\subsection{Number of significant spectra involved}

For a given value of $k$ (number of elementary spectra considered), a matrix of noise can be defined by $B_{k} \approx A-A_{k}$. The value of the standard deviation $\sigma_{k}$ related to $B_{k}$ can then be calculated for different values of $k$. Figure 3 reports these values for the NGC 7023 and $\rho$ Oph-SR3 data. Independently, the value of the noise (standard deviation) can be calculated on the map, either by taking the signal on the faintest pixels (if devoided of spectral information) or by taking difference spectra on pixels carrying identical spectral information. The first case was applied to NGC 7023 and the second to $\rho$ Oph-SR3. The comparison of the value of $\sigma_{k}$ with that of the noise determined on the map allows the number of statistically significant spectra involved in the data reconstruction to be constrained. In the case of NGC 7023, the noise is estimated to be $9.8 \times 10^{-3} \mathrm{Jy}$ per pixel, indicating that $k=4$ is a good estimation of the number of elementary spectra present on the map (cf. Fig. 3). For $\rho$ Oph-SR3, the noise is estimated to be $7.9 \times 10^{-3} \mathrm{Jy}$ per pixel and only $k=3$ elementary spectra are necessary to account for the average information contained in the data. In further studies, it might be possible to search for additional information contained in the other elementary spectra. These spectra may account for weak spectral variations in position and intensity on individual bands.

\subsection{The transformation matrix}

The next step consists of determining the transformation matrix $T$, which allows us to calculate the matrix of elementary chemical spectra $E$ (Eq. (4)) and the matrix of weight coefficients $C$ (Eq. (7)). The matrix $T$ cannot be obtained from mathematical considerations only and physical constraints have to be set. In particular, the presence of negative values in the $C$ and $E$ matrices are not physically consistent and the corresponding solutions have therefore to be excluded. This positivity criterion is objectively justified. In their analysis, Boissel et al. (2001) built the $T$ matrix by manually tuning the parameters. The authors tried to respect as much as possible the positivity criterion and introduced two additional subjective criterions.

(i) The first two elementary spectra $E_{1}$ and $E_{2}$ were supposed to be linear combinations of the first two eigenvectors $V_{1}$ and $V_{2}$ obtained by SVD. This is equivalent to seting four of the sixteen values of $T$ to zero.
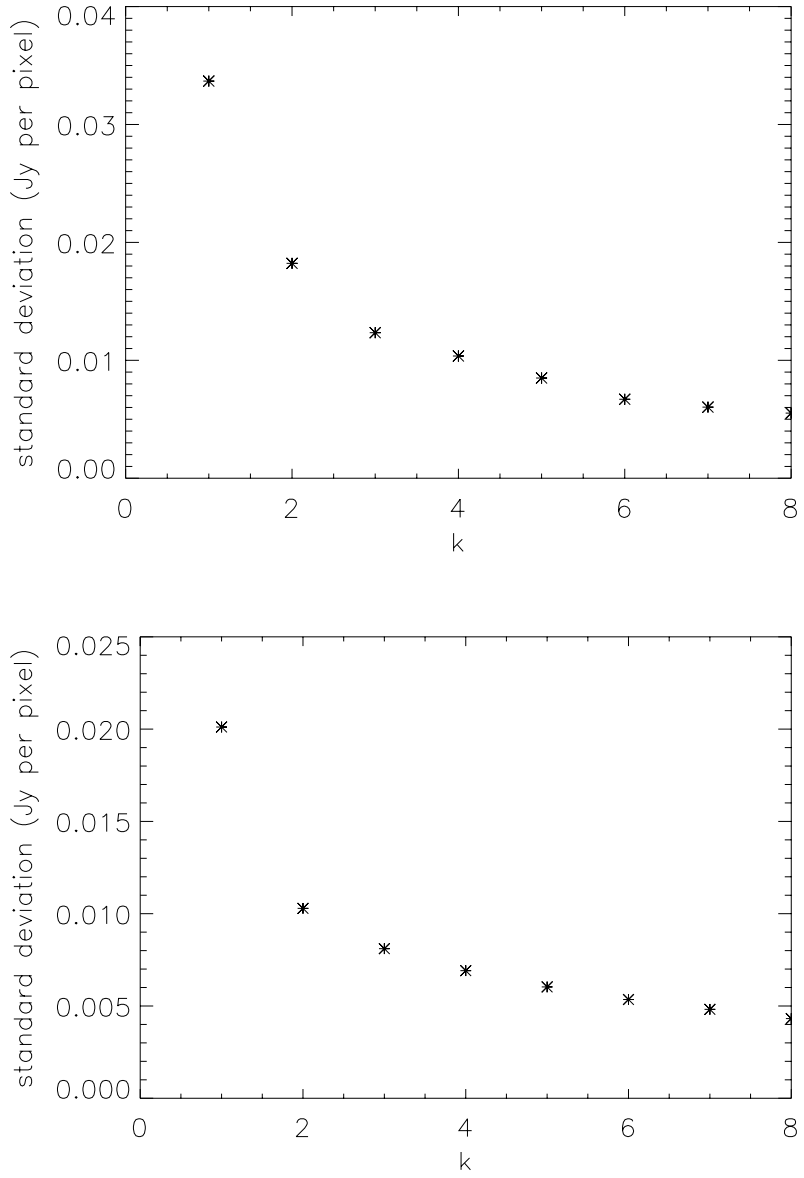

Fig. 3. Standard deviation $\sigma_{k}$ as a function of the number of spectra $k$ which are considered when applying Singular Value Decomposition to the ISOCAM-CVF data of NGC 7023 (upper panel) and of $\rho$ Oph-SR3 (lower panel).

(ii) The spectra $E_{3}$ and $E_{4}$ were built to maximize the spectral and spatial decorrelations between all the spectra.

In the present work, we have dropped all subjective criteria, keeping only the positivity criterion. A $\gamma$ value was defined as the sum of the negative contributions of each spectrum on each pixel:

$\gamma=\sum_{i=1}^{m} \sum_{j=1}^{k} \sum_{l=1}^{n} \min \left[C_{i j} \times E_{j l}, 0\right]$.

The $T$ matrix which maximizes the value of $\gamma$ is the matrix which best fullfills the positivity criterion. Minimizing (or maximizing) a function (here $\gamma$ ) of $\mathrm{p}$ variables (here the $k \times k$ parameters of $T$ ) is a usual problem in physics and many mathematical approaches are possible to solve it. In the present work, a Monte Carlo search method using the Metropolis algorithm has been performed. The chemical spectra and the matrix of weight coefficients are then easily derived from $T$ using Eqs. (4) and (7).

\subsection{Contamination by zodiacal light}

As mentionned previously, we were unable to apply our analysis to the HPHP spectra of NGC 7023 which includes 
substraction of zodiacal light emission. A first difficulty is introduced by the presence of negative values in the data which cannot be accounted for by random noise only. Therefore, to reproduce the data either weight coefficients or values in the elementary spectra have to be negative. This directly affects the value of $\gamma$. The latter was found to be significantly larger when more recently processed (HPDP) data was used. It is certainly not satisfactory to use data contaminated by zodiacal (and straylight) emission. However, the HPDP data in which these contributions are removed happened to be of poorer quality from the point of view of our mathematical analysis. We note that the zodiacal light intensity is of the order of the difference between the scan up and the scan down which are averaged in the ISOCAM data processing. These differences are due to residual in the correction of transient effects. Hopefully, this point could be clarify when higher-quality data will be available for this object (cf. Sect. 7).

\section{Description of the results}

\subsection{Spectra}

The elementary chemical spectra extracted from the SVD-Monte-Carlo analysis are presented in Figs. 4 and 5 for NGC 7023 and $\rho$ Oph-SR3 respectively. Each spectrum has been normalized by its integrated intensity.

Spectrum 4 in Fig. 4 appears as a pure continuum spectrum whithout any obvious spectral features. The jump observed around $9 \mu \mathrm{m}$ coincides with the change from the shortwavelength to the long-wavelength detector in the CVF mode and is due to the uncorrected stray light (Boulanger et al. 2004).

In the analysis of the two objects, two spectra (spectra 1 and 2) present the AIB features usually attributed to PAHs. In their paper, Boissel et al. (2001) presented a mean PAH spectrum and a differential spectrum to trace the variations of the 11.3 and $12.7 \mu \mathrm{m}$ bands relative to the other bands. The new spectra obtained with the SVD-Monte Carlo method reveal two different typical PAH populations which share common characteristics in both objects (cf. Sect. 6.1). An important result is the absence of major continuum emission in these spectra, a result which naturally comes from the analysis without making any assumption.

Spectrum 3 is similar in NGC 7023 and $\rho$ Oph-SR3. It consists of a continuum and bands which are shifted and broader compared with the AIBs. This spectrum is certainly the best achievement of our analysis. A spectral decomposition in lorentzian functions and continua (linear or polynomial) such as discussed by Boulanger et al. (1998) does not allow such information to be derived.

Spectrum 3 is quite different from the mid-IR spectra usually presented in the literature and questions could be raised about its physical validity. Fortunately, this spectrum can be directly compared to the ISOCAM-CVF data of the reflection nebula Ced 201 (Cesarsky et al. 2000). In this object, a few pixels exhibit a spectrum which appears to be similar to calculated spectrum 3 for both objects (Fig. 6). All these spectra exhibit a continuum, broad features centered at 7.8 and $12.4 \mu \mathrm{m}$ and two bands at 6.2 and $11.3 \mu \mathrm{m}$. It is likely that particular physical conditions prevail in Ced 201 which make the carriers of spectrum 3 the dominant emitters. In NGC 7023 and $\rho$ Oph-SR3, spectrum 3 is mixed everywhere with the classical AIBs. The SVD-Monte Carlo analysis is then necessary to disentangle the different spectra. Such analysis was not performed in Ced 201 because of the very limited number of bright pixels in this object.

The agreement with Ced 201 is the strongest point to validate our SVD-Monte Carlo analysis; the extracted spectra, which are mathematical solutions, appear as physically realistic. Even in the case of NGC 7023, in which we were enable to use the HPDP data, the results appear very acceptable. The "spurious" emission, in particular the zodiacal light is likely spread in the extracted spectra. The zodiacal spectrum exhibits continuum emission rising towards large wavelengths (Reach et al. 2000). Such continuum is not apparent in spectra 1 and 2 in Fig. 4. It could be present in spectrum 3 although it is not obvious when compared to the other spectra in Ced 201 and $\rho$ Oph-SR3 (Fig. 6) in which the zodiacal light was substracted. Spectrum 4 seems to be present all over the map (cf. Fig. 4) and is another possibility to carry the zodiacal light in NGC 7023.

\subsection{Spatial distribution}

The maps presented in Figs. 4 and 5 display the relative contribution of each spectrum to the total integrated intensity, the relative contribution of spectrum $j$ at the pixel $i$ is

$$
I_{\mathrm{rel}}(i, j)=\frac{C_{i j} \times \sum_{l=1}^{n} E_{j l}}{\sum_{j=1}^{k} \sum_{l=1}^{n} C_{i j} E_{j l}}
$$

which can be simplified to

$$
I_{\mathrm{rel}}(i, j)=\frac{C_{i j}}{\sum_{j=1}^{k} C_{i j}}
$$

since the spectra are normalized. When increasing distance from the star, the mid-IR emission in the two objects is found to be dominated first by spectrum 1 , then by spectrum 2 and finally by spectrum 3 . Spectrum 4 was only extracted from the NGC 7023 data. It is located extremely close to the star involving a very small number of pixels. In the case of $\rho$ Oph-SR3, it is also present on a few pixels but is more strongly mixed with other components, precluding a proper extraction (cf. spectrum at position 1 in Fig. 2).

Figure 7 displays the relative contribution of the four spectra along two cuts across the northern PDR of NGC 7023 and the PDR of $\rho$ Oph-SR3. These cuts clearly show the sharp edges demarcating the regions dominated by the different spectra. The similarities, in both objects, of the extracted spectra and their relative spatial distributions strongly suggest that the nature of the chemical populations which dominate the IR emission is driven by the local physical conditions. This point is further discussed in the next section. 

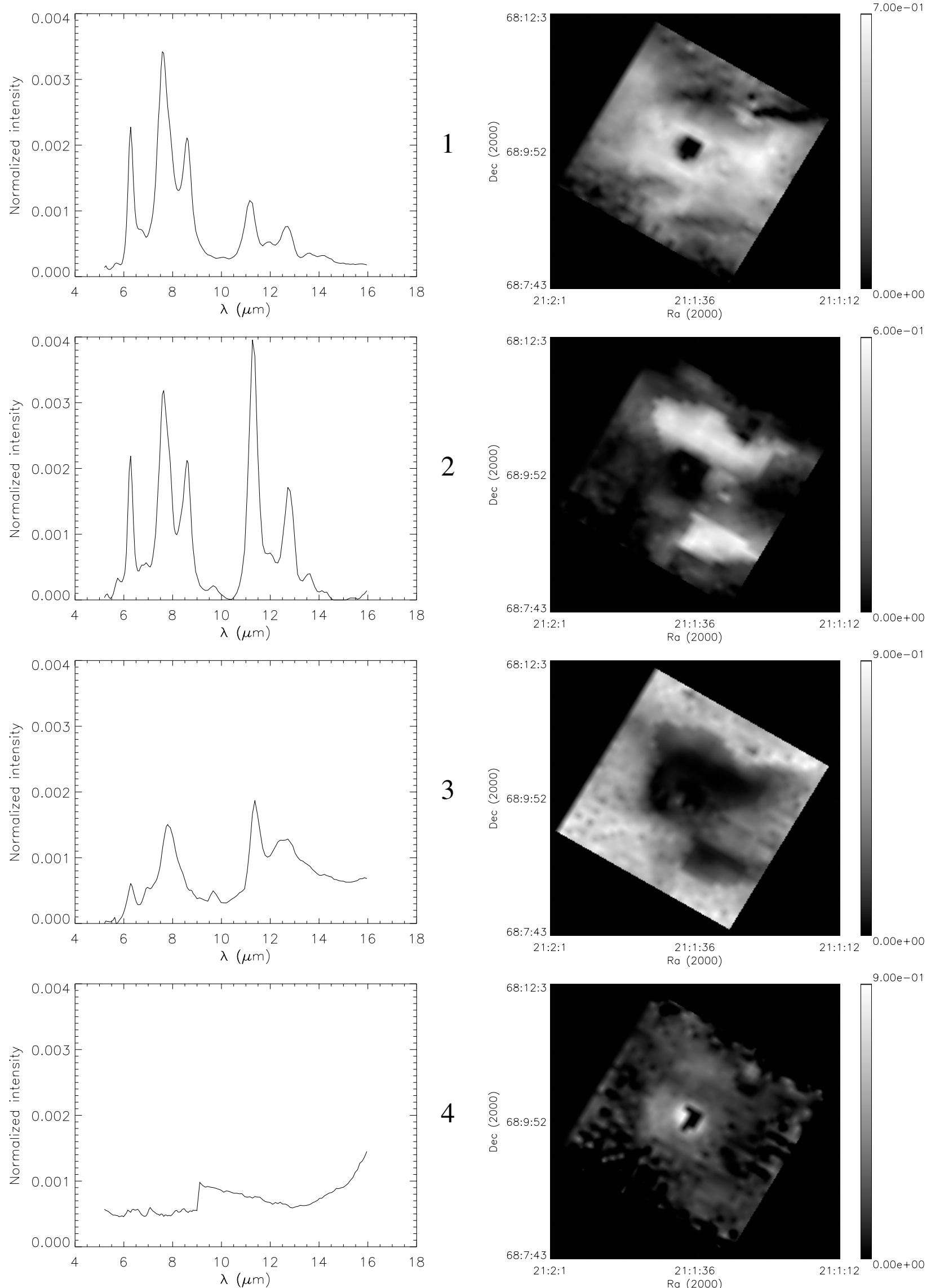

Fig. 4. Results of the SVD-Monte Carlo analysis applied to NGC 7023 data. Left: spectra are normalized by their integrated intensity. Right: relative contribution of each spectrum to the map (cf. Sect. 5). 

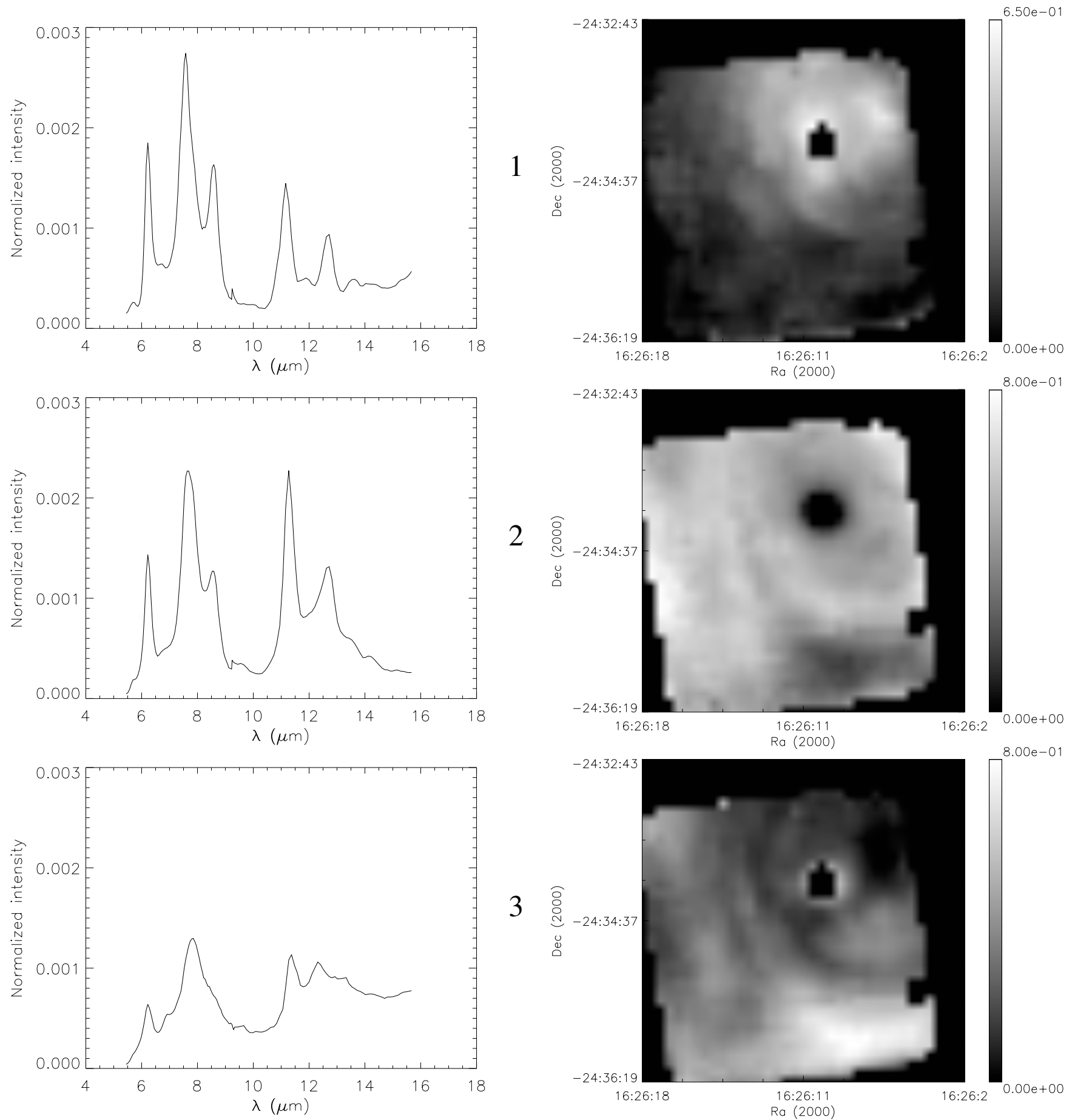

Fig. 5. Results of the SVD-Monte Carlo analysis applied to $\rho$ Oph-SR3 data. Left: spectra are normalized by their integrated intensity. Right: relative contribution of each spectrum to the map (cf. Sect. 5).

\section{Interpretation: PAH molecules and very small grains}

The nature of the various species dominating the mid-IR emission is discussed here using the retrieved spectral and spatial information. We distinguish the contribution from molecular carriers (PAH-like species) from that of larger particles (hot grains at thermal equilibrium and very small grains). The chemical link between the population of very small grains and molecular carriers is emphasized and possible candidates are proposed on this basis.

\subsection{Molecular carriers}

Spectra 1 and 2 in Figs. 4 and 5 display the AIB features and appear in both objects. The absence of major continuum emission is consistent with laboratory spectra on gas-phase PAHs (cf. for instance Joblin et al. 1995). Looking at the 

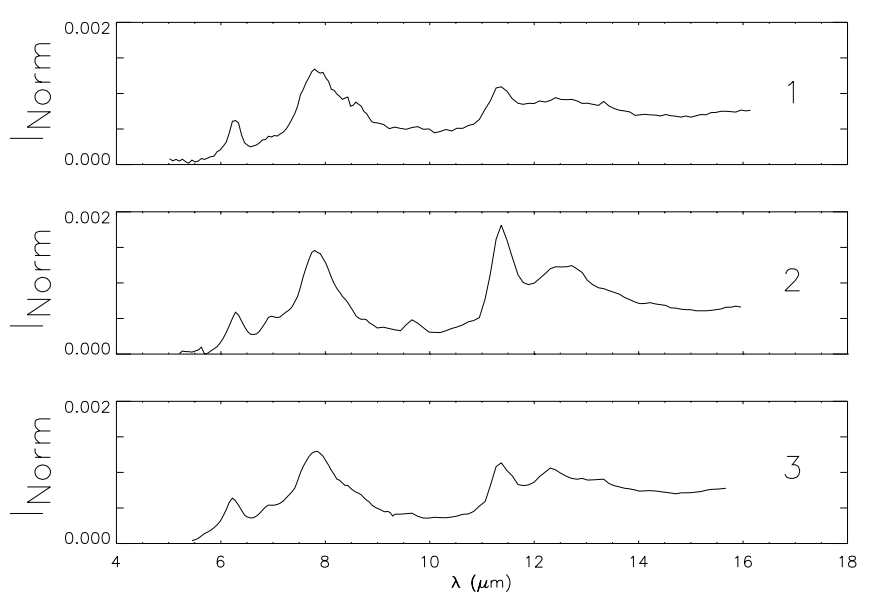

Fig. 6. Upper spectrum (1) is measured in the reflection nebula Ced 201 on one pixel of the ISOCAM map. Lower spectra (2 and 3) are mathematical spectra extracted from our SVD-Monte-Carlo analysis applied to NGC 7023 and $\rho$ Oph-SR3 respectively. Spectra are normalized by their integrated intensity.

spectra, it is clear that there are strong variations in the relative band intensities between the [6-9 $\mu \mathrm{m}]$ and $[11-13 \mu \mathrm{m}]$ ranges. Theoretical (Langhoff 1996 for instance) and laboratory studies (Szczepanski \& Vala 1993; Hudgins \& Allamandola 1995) have shown that the ratio of the $6.2-" 7.7 "-8.6 \mu \mathrm{m}$ to the 11.3-12.7 $\mu \mathrm{m}$ bands increases significantly when PAHs are positively ionized. Using this result, spectrum 1 appears to be dominated by PAH cations and spectrum 2 by PAH neutrals.

In the two maps of NGC 7023 and $\rho$ Oph-SR3 (Figs. 4-5), spectrum 1 is located around the star, whereas spectrum 2 is more significant inside the cloud, implying an increased abundance of PAHs cations closer to the star. This observation is consistent with previous work on the reflection nebula NGC 1333-SVS3 (Joblin et al. 1996; Sloan et al. 1999), which shows a decrease of the $8.6 / 11.3 \mu \mathrm{m}$ intensity ratio when increasing the distance from the star. The charge balance results from an equilibrium between photoionization and electronic recombination rates. Considering the combined effect of UV flux dilution whith increasing distance from the star and of higher electron density at the surface of the dense molecular cloud, an increased abundance of neutral PAHs can be qualitatively explained.

An interesting point is the variation of the band positions between the spectra of $\mathrm{PAH}$ cations $\left(\mathrm{PAH}^{+}\right)$and neutrals $\left(\mathrm{PAH}^{0}\right)$ (see Table 1 and Fig. 8). The most significant variation is for the $11.3 \mu \mathrm{m}$ band which peaks at $11.16 \mu \mathrm{m}$ in the $\mathrm{PAH}^{+}$spectrum and $11.27-11.31 \mu \mathrm{m}$ in the $\mathrm{PAH}^{0}$ spectrum. This shift is real. We have found that the positions of the $0-0 \mathrm{~S}(5), 0-0 \mathrm{~S}(3)$ and $0-0 \mathrm{~S}(2) \mathrm{H}_{2}$ lines at 6.909, 9.665 and $12.279 \mu \mathrm{m}$ respectively are accurate to better than $0.03 \mu \mathrm{m}$ in the CVF spectra. The variation of the $11.3 \mu \mathrm{m}$ peak position by $0.13 \mu \mathrm{m}\left(10 \mathrm{~cm}^{-1}\right)$ appears as a global shift of the band without clear change of the band width. This suggests that ionization leads to a $10 \mathrm{~cm}^{-1}$ blue-shifted line. Another alternative is to calculate the differential spectrum between the $\mathrm{PAH}^{+}$and $\mathrm{PAH}^{0} 11.3 \mu \mathrm{m}$ bands when normalized to by their integrated intensity. This leads to a positive band at $11.0 \mu \mathrm{m}$ and a negative band at $11.47 \mu \mathrm{m}$. In this case a shift of $0.47 \mu \mathrm{m}\left(30 \mathrm{~cm}^{-1}\right)$ is found between the $11.0 \mu \mathrm{m}$ attributed to cations and the $11.47 \mu \mathrm{m}$ attributed to the same molecules in their neutral form. It is interesting to mention that this shift is exactly the one obtained in laboratory experiments for non adjacent $\mathrm{CH}$ (Hudgins \& Allamandola 1999). The authors have also reviewed the observations showing the presence of a weaker band at $11.0 \mu \mathrm{m}$ which they attribute to PAH cations of medium size (fewer than 50 carbon atoms) which contain solo and therefore non adjacent $\mathrm{CH}$ groups. Finally, our data analysis was restricted to the $[5.2-15.9 \mu \mathrm{m}]$ range whereas the ISOCAM data is available up to $16.5 \mu \mathrm{m}$. It therefore covers part of the $16.4 \mu \mathrm{m}$ band which has been attributed to interstellar PAHs (Moutou et al. 2000; Van Kerckhoven et al. 2000). Looking at the spatial distribution of the $16.4 \mu \mathrm{m}$ band, we found it to be correlated with the distribution of PAH neutrals. This does not mean that PAH cations do not emit at these wavelengths but their contribution is minor.

\subsection{Continuum and emission from grains}

The presence of a steep continuum at long wavelengths (spectrum 4) and located close to the star (Figs. 1, 2 and 4) is characteristic of hot grain emission. In the case of NGC 7023, the presence of weak continuum emission over the whole nebula as seen on Map 4 in Fig. 4 is due to the uncorrected zodiacal and stray light emission (Boulanger et al. 2004).

Another type of continuum emission is present in PDRs far from exciting sources, as emphasized by Abergel et al. (2002) in the southern part of Orion B (NGC 2023, NGC 2024, L1630). This continuum emission has been reported several years ago and proposed to be associated with the AIBs spectrum but not directly correlated with the bands (Cohen et al. 1985, 1986, 1989; Roche et al. 1989; Bregman et al. 1989). The analysis which has been performed here reveals the spectral features which are associated with this continuum (cf. spectrum 3 in Figs. 4-5). The similarities of spectrum 3 for the two objects suggest that carriers from the same chemical family are involved. Very interesting is the strong resemblance with the spectrum of Ced 201 (Fig. 6). Cesarsky et al. (2000) attributed the latter spectrum to Very Small carbonaceous Grains (VSGs). The authors also suggested that these VSGs transform into the carriers of the AIBs under the action of the UV field and/or shock waves. They proposed that such grains could be present everywhere in the interstellar medium but only detected in the mid-IR under special circumstances (for instance in the environment of Ced 201). We report here their clear detection in two additional reflection nebulae, a detection which was made possible by our SVD-Monte Carlo method.

It is interesting to focus on the broad band observed around $7.8 \mu \mathrm{m}$ in the two VSG spectra. Lorentzian decompositions of ISO Short-Wavelength-Spectrometer (SWS) spectra revealed the presence of at least three components in the " $7.7 \mu$ m" massif: 7.5, 7.6 and $7.8 \mu \mathrm{m}$ according to Joblin et al. 2000 and Verstraete et al. 2001. Peeters et al. (2002) studied a large number of spectra showing that the two main bands are located at 7.6 and $7.8 \mu \mathrm{m}$. The authors discussed the fact that 
Table 1. Positions and relative intensities for the five AIBs observed in the deconvolved spectra and for adjacent continuum. Intensities are normalized relative to the "7.7" $\mu \mathrm{m}$ band intensity.

\begin{tabular}{|c|c|c|c|c|c|c|c|c|c|c|c|c|}
\hline & \multicolumn{10}{|c|}{ Bands } & \multicolumn{2}{|c|}{ Continuum } \\
\hline & \multicolumn{2}{|c|}{$6.2 \mu \mathrm{m}$} & \multicolumn{2}{|c|}{ "7.7" $\mu \mathrm{m}$} & \multicolumn{2}{|c|}{$8.6 \mu \mathrm{m}$} & \multicolumn{2}{|c|}{$11.3 \mu \mathrm{m}$} & \multicolumn{2}{|l|}{$12.7 \mu \mathrm{m}$} & \multirow{2}{*}{$\begin{array}{l}10 \mu \mathrm{m} \\
I_{r \lambda_{0}}\end{array}$} & \multirow{2}{*}{$\begin{array}{l}15 \mu \mathrm{m} \\
I_{r \lambda_{0}}\end{array}$} \\
\hline & $\lambda_{0}$ & $I_{r \lambda_{0}}$ & $\lambda_{0}$ & $I_{r \lambda_{0}}$ & $\lambda_{0}$ & $I_{r \lambda_{0}}$ & $\lambda_{0}$ & $I_{r \lambda_{0}}$ & $\lambda_{0}$ & $I_{r \lambda_{0}}$ & & \\
\hline \multicolumn{13}{|l|}{ NGC 7023} \\
\hline $\mathrm{PAH}^{+}$ & 6.27 & 0.66 & 7.57 & 1.00 & 8.59 & 0.62 & 11.16 & 0.34 & 12.7 & 0.22 & 0.08 & 0.06 \\
\hline $\mathrm{PAH}^{0}$ & 6.27 & 0.67 & 7.63 & 1.00 & 8.59 & 0.67 & 11.31 & 1,25 & 12.7 & 0.80 & 0.02 & 0.01 \\
\hline VSG & 6.27 & 0.41 & 7.80 & 1.00 & - & - & 11.37 & 1.24 & $12.4-12.7$ & 0.85 & 0.21 & 0.42 \\
\hline \multicolumn{13}{|l|}{$\rho$ Oph } \\
\hline $\mathrm{PAH}^{+}$ & 6.22 & 0.67 & 7.57 & 1.00 & 8.58 & 0.59 & 11.16 & 0.54 & 12.72 & 0.35 & 0.07 & 0.15 \\
\hline $\mathrm{PAH}^{0}$ & 6.22 & 0.63 & 7.63 & 1.00 & 8.63 & 0.56 & 11.27 & 1.00 & 12.72 & 0.57 & 0.11 & 0.12 \\
\hline VSG & 6.22 & 0.49 & 7.85 & 1.00 & - & - & 11.36 & 0.87 & 12.30 & 0.82 & 0.27 & 0.577 \\
\hline
\end{tabular}
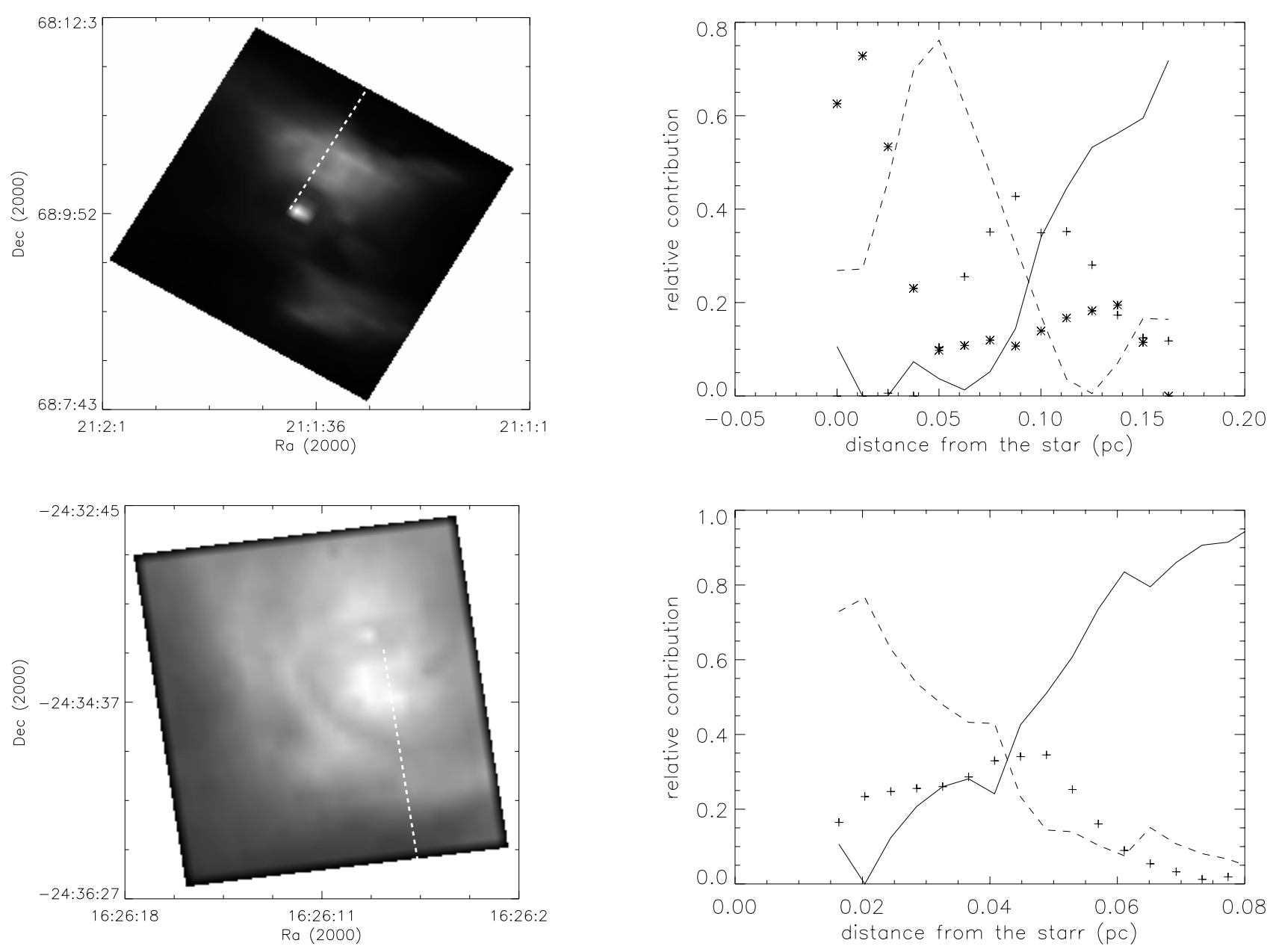

Fig. 7. Left: cuts across the northern PDR of NGC 7023 and across the PDR of $\rho$ Oph-SR3. Right: relative contribution along these cuts of the different components extracted from our analysis (Figs. 4 and 5) including spectra 1 (dashed line), 2 (crosses), 3 (solid line) and 4 (stars).

the $7.6 \mu \mathrm{m}$ band is well understood by experimental studies and quantum chemical calculations on PAHs but the $7.8 \mu \mathrm{m}$ subfeature remains an enigma. In the present study (see Table 1 and Fig. 8), we found that the band peaking around $7.6 \mu \mathrm{m}$ is carried by neutral and ionised PAH whereas the broad band around $7.8 \mu \mathrm{m}$ is carried by VSGs. The shift of $0.1 \mu \mathrm{m}$ reported by Peeters et al. (2002) for the $6.2 \mu \mathrm{m}$ band is not evidenced by our analysis. We found that the VSG spectrum lacks the $8.6 \mu \mathrm{m}$ band and its $12.7 \mu \mathrm{m}$ band is not prominent but is included in a plateau extending from 12 to $14 \mu \mathrm{m}$. Finally, the $11.3 \mu \mathrm{m}$ band is found to be red-shifted to $11.4 \mu \mathrm{m}$.

The spatial distribution that was derived for spectrum 3 attributed to carbonaceous VSGs shows that their emission is present only in the molecular cloud and that there is a sharp 


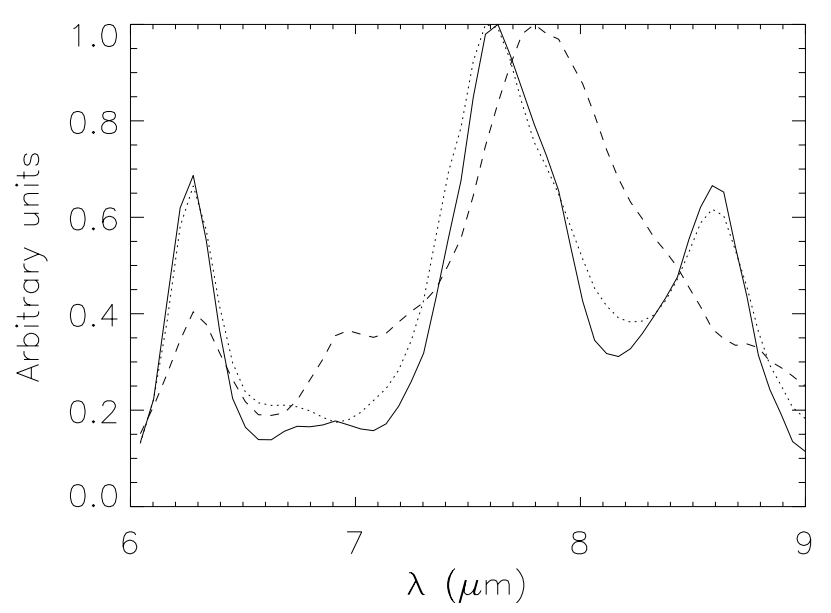

Fig. 8. The 6 to $9 \mu \mathrm{m}$ range of the $\mathrm{PAH}^{+}$(dotted line), $\mathrm{PAH}^{0}$ (solid line) and VSG (dashed line) spectra extracted from the NGC 7023 data.

edge (decrease of contribution) where the neutral PAH emission dominates the observed spectrum (Fig. 7). This clearly points to a chemical link between PAHs and carbonaceous VSGs. Free-flying PAHs appear to be generated when carbonaceous VSGs are destroyed. This strongly reinforces the idea that PAHs are produced by photoevaporation of larger grains as was previously proposed to account for the strong spatial variations observed in the IRAS $12 / 100 \mu \mathrm{m}$ ratio at the surface of molecular clouds (Boulanger et al. 1990; Bernard et al. 1993).

\subsection{Properties of carbonaceous VSGs}

The size of very small grains experiencing stochasting heating can be derived from the knowledge of their temperature, internal energy and heat capacity. This has been calculated for very small carbonaceous (PAH-like) grains by Draine \& Li (2001).

A crude estimate of the temperature of carbonaceous VSGs was obtained as follows. The shape of spectra 3 indicates that the maximum of emission for the VSGs is located at wavelengths longer than $11 \mu \mathrm{m}$ which corresponds, in the black body approximation, to temperatures lower than $270 \mathrm{~K}$. The UV flux in the region of maximum emission for the VSGs was calculated using an updated version of the PDR model of Le Bourlot et al. (1993), which is publically available at http://www.aristote.biophy.jussieu.fr/MIS (see also Le Petit et al. 2002) This is a stationary one-dimensional plan parallel model which consistently treats the chemistry, the thermal balance and the UV radiative transfer. It provides a value for the UV flux $F_{\lambda}(\lambda)$ in $\mathrm{W} \mathrm{m}^{-2} \mu \mathrm{m}^{-1}$ for each point inside the cloud. One input of the model is the UV flux impinging the cloud. This was calculated using the synthetic stellar spectra by Kurucz (1991) with a gravity of 4.0 and effective temperatures of $20000 \mathrm{~K}$ in the case of HD 200775 (van den Ancker et al. 1997) and $13000 \mathrm{~K}$ for SR3 (assumed to be a normal B7 star). The surface of the molecular cloud has been defined as the region where PAH cations and neutrals give an equal contribution to the total emission (cf. Fig. 7). The dilution factor was then calculated with a distance between the star and the cloud of $0.083 \mathrm{pc}$ and $0.043 \mathrm{pc}$ in the case of NGC 7023 and $\rho$ Oph-SR3 respectively. Values for the integrated UV flux $(6-13.6 \mathrm{eV})$ are 11400 (NGC 7023) and 763 ( $\rho$ Oph-SR3) in units of $G_{\mathrm{o}}\left(1 G_{\mathrm{o}}=1.6 \times 10^{-10} \mathrm{~W} \mathrm{~cm}^{-2}\right.$; Habing 1968)

Another input of the model is the hydrogen density, $n_{\mathrm{H}}$. To constrain this density, we have used the information on the $0-0 \mathrm{~S}(3) \mathrm{H}_{2}$ line at $9.7 \mu \mathrm{m}$. The emission line attains a maximum at a distance of $0.112 \mathrm{pc}$ from the star in the northern PDR of NGC 7023 (0.057 pc in the case of $\rho$ Oph-SR3), which can be reproduced in the PDR model with $n_{\mathrm{H}}=3 \times 10^{4} \mathrm{~cm}^{-3}$ $\left(n_{\mathrm{H}}=4.5 \times 10^{3} \mathrm{~cm}^{-3}\right)$ We now consider that a VSG with an absorption cross-section $\sigma(\lambda)$ absorbs a photon. The probability that this photon falls in the range $[\lambda, \lambda+\Delta t \lambda]$ is given by $p(\lambda) \mathrm{d} \lambda$. This probability corresponds to the number of photons absorbed during a long period $\Delta t$ divided by the total number of photons absorbed over the same period:

$$
p(\lambda) \mathrm{d} \lambda=\frac{F_{\lambda}(\lambda) \sigma(\lambda)\left(\frac{h c}{\lambda}\right)^{-1} \Delta t \mathrm{~d} \lambda}{\int_{\lambda} F_{\lambda}(\lambda) \sigma(\lambda)\left(\frac{h c}{\lambda}\right)^{-1} \Delta t \mathrm{~d} \lambda}
$$

The mean energy of the absorbed photons can then be expressed as:

$$
\left\langle E_{\mathrm{UV}}\right\rangle=\int_{\lambda} p(\lambda)\left(\frac{h c}{\lambda}\right) \mathrm{d} \lambda=\frac{\int_{\lambda} F_{\lambda}(\lambda) \sigma(\lambda) \mathrm{d} \lambda}{\int_{\lambda} F_{\lambda}(\lambda) \sigma(\lambda)\left(\frac{h c}{\lambda}\right)^{-1} \mathrm{~d} \lambda} .
$$

Since the UV absorption cross-section of VSGs is not known, we have used the mean value measured on gas-phase PAHs (Joblin et al. 1992), which is proportional to the number of carbon atoms $\sigma=N_{\mathrm{C}} \times \sigma_{\mathrm{c}}^{\mathrm{PAH}}$. The mean value of $\left\langle E_{\mathrm{UV}}\right\rangle$ was calculated in the region where the VSG emission is dominant. It falls in the [8.0-8.3] and [7.4-7.6] eV range in NGC 7023 and $\rho$ Oph-SR3 respectively. It has been shown, for PAHs, that a mean emission temperature can be defined when the molecule has lost about half of the absorbed UV energy (Léger et al. 1989). Using this result, this means that the maximum temperature of $270 \mathrm{~K}$ determined above is associated to VSGs with an internal energy larger than $7.4 / 2=3.7$ and $8.0 / 2=4.0 \mathrm{eV}$ in the case of $\rho$ Oph-SR3 and NGC 7023 respectively. This corresponds to a minimum size of about $9.7 \AA$ in $\rho$ Oph-SR 3 and $9.9 \AA$ in NGC 7023, giving a number of carbon atoms larger than 425 and 460 (cf. Fig. 7 from Draine \& Li 2001). These minimum sizes are intermediate between molecules and grains and this is consistent with the presence of bands and continuum in the corresponding spectrum. In order to understand the evolution of the VSG emission with the UV field properties, we have represented in Fig. 9 the intensity of this emission, the mean absorbed energy $\left\langle E_{\mathrm{UV}}\right\rangle$ and the UV absorption power as a function of depth inside the cloud $\left(P_{\mathrm{UV}}=\int_{6}^{13.6 \mathrm{eV}} \sigma F_{\lambda} \mathrm{d} \lambda\right)$. In the case of $\rho$ Oph-SR3, $\left\langle E_{\mathrm{UV}}\right\rangle$ is nearly constant all over the cloud whereas it shows more variation in NGC 7023. The intensity of the VSG emission is expected to follow the UV absorption power. However, Fig. 9 shows that there is a break in the VSG emission when going toward the edge of the cloud. This break shows a variation in the abundance of VSGs. This effect occurs for an absorption power larger than $10^{-24}$ and $4 \times 10^{-25} \mathrm{~W} \mathrm{C}^{-1}$ in the case of NGC 7023 and $\rho$ Oph-SR3 respectively. This strongly suggests that the involved mechanism is photodissociation. It seems consistent to find the largest 

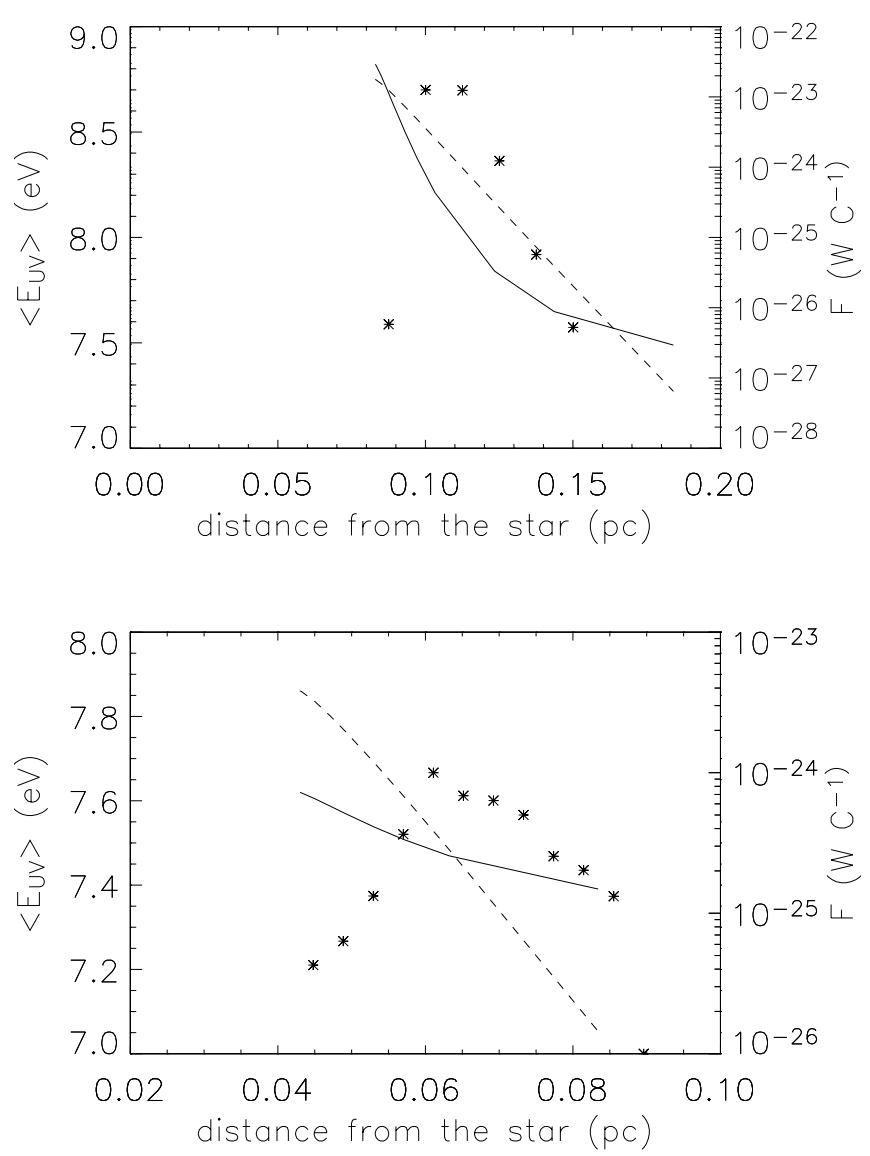

Fig. 9. Intensity of the VSG emission (stars), mean UV energy absorbed by these grains (solid line) and associated UV absorption power (dashed line). Upper pannel corresponds to the northern PDR of NGC 7023 and lower one to $\rho$ Oph-SR3.

dissociation absorption power in NGC 7023 where the VSGs minimum size is the largest.

\subsection{A chemical scenario: Photoprocessing of VSGs and PAHs}

The following scenario is proposed to interpret the results of our SVD-Monte Carlo analysis. Inside the molecular cloud, PAH molecules are trapped in carbonaceous VSGs. A possibility is that these VSGs are PAH clusters. At the edge of the cloud, the clusters are producing free-flying neutral PAHs by photo-evaporation. The evaporation process leads to significant destruction of the VSGs at an absorption power of about $10^{-24} \mathrm{~W} \mathrm{C}^{-1}$. Around the star, PAHs are found to be mainly ionized. This is likely due to the presence of a cavity of lower density gas around the star formed during its formation process (cf. Fuente et al. 2001 in the case of NGC 7023 / HD 200775). In such region, the combination of the lower electron density with a higher photo-ionisation rate leads to an enhancement of the ionized to neutral PAH abundance ratio.

\section{Conclusion and perspectives}

We have illustrated in this paper how a combined SVDMonte Carlo analysis can be used to interpret the mid-IR spectro-imagery data of PDRs. We have been able to show the presence of four populations of emitters: hot grains close to the star, PAH molecules both in their ionized and neutral forms, and carbonaceous VSGs. The analysis allows a full 5-16 $\mu \mathrm{m}$ spectrum and the associated spatial distribution to be derived for each population. The variation of the charge state of PAHs within extended objects is clearly shown for the first time. Another important result is the attribution of the $7.8 \mu \mathrm{m}$ subfeature of the "7.7 $\mu \mathrm{m}$ " massif to the carbonaceous VSGs. These VSGs, which appear as the progenitors of free PAHs, suffer from significant photodissociation at an absorption power of about $10^{-24} \mathrm{~W} \mathrm{C}^{-1}$ and could be used to trace the UV flux in PDRs. The presence of VSGs as an important dust component has been proposed by several authors. Désert et al. (1990) have described them as the carriers of the $25 \mu \mathrm{m}$ emission measured by the Infrared Astronomical Satellite (IRAS). The authors suggested that these VSGs were carbon-dominated but could not prove it. They also attributed the absorption bump at $220 \mathrm{~nm}$ to this population. At this stage, it is not clear how the VSGs of Désert et al. (1990) are related to the carbonaceous VSGs studied in this paper because of the lack of information at $\lambda>16 \mu \mathrm{m}$. Interestingly, Fuente et al. (1992) have shown that the $25 \mu \mathrm{m}$ emission in reflection nebulae exhibits a peculiar behaviour. They proposed that their carriers are small grains easily destroyed by UV radiation. To make a chemical link between these VSG populations, it is therefore very important to extend the present studies to longer wavelengths. This is now becoming possible thanks to the capabilities of the Spitzer Space Telescope (SST). We also expect that the higher sensitivity of the SST will allow to extand our studies on a larger sample of objects and to confirm our results with better-quality data in the case of NGC 7023 as discussed in Sect. 4.3. One important concern is the geometry of the objects in particular when observations have to be interpretated qualitatively with a PDR model. In this paper, it was not necessary to go into such complications but this has to be kept in mind for future studies.

Finally, the information obtained on the new population of carbonaceous VSGs provides guidelines for experimental and theoretical studies. Studies are now in progress to test the possibility that they could be PAH clusters (Rapacioli et al. 2004).

Acknowledgements. We thank J. Le Bourlot for his kind and efficient support for the PDR model and D. Cesarsky for providing his reduced data of NGC 7023 and Ced 201. We thank the anonymous referee for useful comments that improved the quantitative description of the spectra. We also thank F. Boulanger and R. Lorente for sharing their expertise in ISOCAM data reduction.

\section{References}

Abergel, A., Bernard, J. P., Boulanger, F., et al. 2002, A\&A, 389, 239 Allamandola, L. J., Tielens, A. G. G. M., \& Barker, J. R. 1985, ApJ, 290, L25

Banisaukas, J., Szczepanski, J., \& Vala, M. 2004, J. Phys. Chem. A, 108,3713

Bauschlicher, C. W. Jr. 2002, ApJ, 564, 782

Bernard, J.-P., Boulanger, F., \& Puget, J. L. 1993, A\&A, 277, 609

Boissel, P., Joblin, C., \& Pernot, P. 2001, A\&A, 373, L5 
Bontemps, S., André, P., Kaas, A., et al. 2001, A\&A, 372, 173

Boulanger, F., Boissel, P., Cesarsky, D., \& Ryter, C. 1998, A\&A, 339, L194

Boulanger, F., Falgarone, E., Puget, J. L., \& Helou, G. 1990, ApJ, 364, 136

Boulanger, F., Lorente, R., Miville Deschênes, M. A., et al. 2004, A\&A, submitted

Bregman, J. D., Allamandola, L. J., Witteborn, F. C., Tielens, A. G. G. M., \& Geballe, T. R. 1989, ApJ, 344, 791

Cesarsky, D., Lequeux, J., Abergel, A., et al. 1996, A\&A, 315, L305

Cesarsky, D., Lequeux, J., Ryter, C., \& Gerin, M. 2000, A\&A, 354, L87

Chokshi, A., Tielens, A. G. G. M., Werner, M. W., \& Castelaz, M. W. 1988, ApJ, 334, 803

Cohen, M., Allamandola, L., Tielens, A. G. G. M., et al. 1986, ApJ, 302, 737

Cohen, M., Tielens, A. G. G. M., \& Allamandola, L. J. 1985, ApJ, 299, L93

Cohen, M., Tielens, A. G. G. M., Bregman, J., et al. 1989, ApJ, 341, 246

Cook, D. J., Schlemmer, S., Balucani, N., et al. 1998, J. Phys. Chem. A, 102, 1465

de Frees, D. J., Miller, M. D., Talbi, D., Pauzat, F., \& Ellinger, Y. 1993, ApJ, 408, 530

Désert, F.-X., Boulanger, F., \& Puget, J.-L. 1990, A\&A, 237, 215

Draine, B. T., \& Li, A. 2001, ApJ, 551, 807

Ellinger, Y., Pauzat, F., \& Lengsfield, B. H. 1999, J. Mol. Struct., 458, 203

Federman, S. R., Knauth, D. C., Lambert, D. L., \& Anderson, B. G. 1997, ApJ, 489, 758

Fuente, A., \& Martin-Pintado, J. 1997, ApJ, 477, L107

Fuente, A., Martin-Pintado, J., Cernicharo, J., Brouillet, N., \& Duvert, G. 1992, A\&A, 260, 341

Fuente, A., Neri, R., Martin-Pintado, J., et al. 2001, A\&A, 366, 873

Gerin, M., Phillips, T. G., \& Keene, J., et al. 1998, ApJ, 500, 329

Habing, H. J. 1968, BAN, 421

Hudgins, D. M., \& Allamandola, L. J. 1995, J. Phys. Chem., 99, 3033

Hudgins, D. M., \& Allamandola, L. J. 1999, ApJ, 516, L41

Hudgins, M., Bauschlicher, C. W. Jr., \& Allamandola, L. J. 2001, Mol. \& Biomol. Spec., 57, 907

Hudgins, D. M., Sandford, S. A., \& Allamandola, L. J. 1994, J. Phys. Chem., 98, 4243

Huneycutt, A. J., Casaes, R. N., Saykally, R. J., Chung, C.-Y., \& Lee, Y.-P. 2004, Chem. Phys. Chem., 5, 321

Joblin, C., Abergel, A., \& Bregman, J. 2000, in ISO beyond the peaks, ESA, Pub. Ser., SP-456, 49

Joblin, C., Boissel, P., Léger, A., d'Hendecourt, L., \& Défourneau, D. 1995, A\&A, 299, 835

Joblin, C., Léger, A., \& Martin, P. 1992, ApJ, 393, L79
Joblin, C., Tielens, A. G. G. M., Geballe, T. R., \& Wooden, D. H. 1996, ApJ, 460, L119

Kessler, M. F., Steinz, J. A., Anderegg, M. E., et al. 1996, A\&A, 315, L27

Kim, H.-S., Wagner, D. R., \& Saykally, R. J. 2001, Phys. Rev. Lett., 86,5691

Kurtz, J. 1993, A\&A, 255, L1

Kurucz, R. L. 1991, BAAS, 23, 1047

Langhoff, S. R. 1996, J. Phys. Chem., 100, 2819

Le Bourlot, J., Pineau des Forêts, G., Roueff, E., \& Flower, D. R. 1993, A\&A, 267, L233

Léger, A., d'Hendecourt, L., \& Défourneau, D. 1989, A\&A, 216, 148

Léger, A., \& Puget, J.-L. 1984, A\&A, 137, L5

Léger, M. N., \& Wentzell, P. D. 2002, Chemometrics and Intelligent Laboratory System, 62, 171

Lemaire, J. L., Field, D., \& Maillard, J. P. 1999, A\&A, 349, 253

Le Petit, F., Roueff, E., \& Le Bourlot, J. 2002, A\&A, 390, 309

Laureijs, R. J., Acosta-Pulido, J., Abraham, P., et al. 1996, A\&A, 315, L313

Martini, P., Sellgren, K., \& Hora, J. L. 1997, ApJ, 484, 296

Mattioda, A. L., Hudgins, D. M., Bauschlicher, C. W. Jr., Rosi, M., \& Allamandola, L. J. 2003, J. Phys. Chem. A, 107, 1486

Moutou, C., Verstraete, L., Léger, A., Sellgren, K., \& Schmidt, W. 2000, A\&A, 354, L17

Pauzat, F., \& Ellinger, Y. 2002, Chem. Phys., 280, 267

Pauzat, F., Talbi, D., \& Ellinger, Y. 1995, A\&A, 293, 263

Peeters, E., Hony, S., \& Van Kerckhoven, C. 2002, A\&A, 390, 1089

Murakami, H., Freund, M. M., Ganga, K., et al. 1996, Publ. Astron. Soc. Japan, 48, L41

Oomens, J., van Roij, A. J. A., Meijer, G., \& von Helden, G. 2000, ApJ, 542, 404

Rapacioli, M., Calvo, F., Spiegelman, F., Joblin, C., \& Wales, D. J., submitted

Reach, W. T., Boulanger, F., Contursi, A., \& Lequeux, J. 2000, A\&A, 361,895

Roche, P. F., Aitken, D. K., \& Smith, C. H. 1989, MNRAS, 236, 485

Rogers, C., Heyer, M. H., \& Dewdney, P. E. 1995, ApJ, 442, 694

Sellgren, K., Allamandola, L. J., Bregman, J. D., Werner, M. W., \& Wooden, D. H. 1985, ApJ, 299, 416

Sellgren, K., Werner, M. W., \& Dinerstein, H. L. 1992, ApJ, 407, 238

Sloan, G. C., Hayward, T. L., Allamandola, L. J., et al. 1999, ApJ, 513, L65

Szczepanski, J., \& Vala, M. 1993, ApJ, 414, 646

Van Kerckhoven, C., Hony, S., Peeters, E., et al. 2000, A\&A, 357, 1013

van den Ancker, M. E., Thé, P. S., Tjin A Djie, H. R. E., et al. 1997, A\&A, 324, L33

Verstraete, L., Pech, C., Moutou, C., et al. 2001, A\&A, 372, 981

Witt, A. N., Petersohn, J. K., \& Bohlin, R. C. 1992, ApJ, 395, L5 